\title{
Crosshole seismic tomography including the anisotropy effect
}

\author{
Ying Rao ${ }^{1,2}$ and Yanghua Wang ${ }^{1}$ \\ ${ }^{1}$ Centre for Reservoir Geophysics, Department of Earth Science and Engineering, Imperial College London, UK \\ ${ }^{2}$ State Keylab of Petroleum Resource \& Prospecting, China Unversity of Petrolem (Beijing), China
}

\begin{abstract}
Velocity anisotropy caused by fine-layering makes strong effect to the travel path with large vertical offset in crosshole seismic data. This effect must be considered in seismic waveform tomography, so that the inversion procedure can match the waveforms in real data. In this paper, we proposal to properly take account this anisotropic effect in two stages. First, we invert for the anisotropy parameter simultaneously with the (horizontal) velocity in travel time tomography. Then we incorporate this estimated anisotropy parameter model in waveform simulation for waveform tomography, to refine the velocity image. When considering this anisotropic effect, far-offset data are properly used in the inversion, then both travel time and waveform tomography may have a better ray coverage, producing a velocity image with a better resolution.
\end{abstract}

Keywords: crosshole seismic, tomography, velocity anisotropy

\section{Introduction}

Sedimentary rocks with a layered sequence can cause a most common type of velocity anisotropy, vertical transverse isotropy (Thomsen 1986). This type of anisotropy makes stronger effect to the travel path with large source-receiver offset. In this paper, we investigate this velocity anisotropy effect in the crosshole seismic data between two vertical boreholes. We estimate the anisotropy parameter by travel time tomography, and then incorporate this anisotropy model in waveform tomography that follows.

Travel time tomography is a cost-effective way to determine the velocity distribution in the research area between two parallel boreholes. It uses the first arrival times to reconstruct the velocity model. It is also an ideal option to build a reliable initial model for the following waveform tomography. However, the large-offset data often appear to be incompatible with the near-offset data available, and cause significant numerical artifact in velocity reconstruction. The influence of the large-offset travel time data in the inversion can be reduced pragmatically by set a data-related covariance matrix in the least-squares formula (Rao and Wang 2005). By down-weighting far-offset data and assuming the media be isotropic, travel time inversion effectively generates the image of horizontal velocity.

However, large-offset data are necessary for an inversion to constrain the lateral variation of the subsurface velocity field. Furthermore, wave propagates in a layered sequence of different media (isotropic or not) just as propagating through an anisotropic medium (Backus 1962, Thomsen 1986). To effectively use all-offsets data available, we attempt to invert also the anisotropy parameter in travel time inversion. For crosshole seismic, Chapman and Pratt (1992) and Pratt and Chapman (1992) developed linear systems for 2-D travel time 
tomography in anisotropic media, and Pratt et al. (1993) and Pratt and Sams (1996) showed that the anisotropic velocity tomography is a valuable tool to detect the discontinuities in the investigation region. Zhou et al. (2008) used a nonlinear inversion method for Thomsen's anisotropic parameters from the travel time inversion.

For waveform tomography in crosshole geometry, ignoring the existence of anisotropy also causes artefacts in the velocity image. Wang and Rao (2006) applied a weighting taper to the data, related to source-receiver (vertical) offsets, and effectively suppressed the common " $X$ " type artefacts in the velocity image. Pratt and Shipp (1999) considered the anisotropy as a constant only in the background and preset it as a constant shrink factor in the whole research area during the numerical calculation. In this paper, however, we use a 2D anisotropy model effectively in waveform simulation, rather than a simple constant shrinking factor.

In summary, taking account the anisotropic effect, we use far-offset data properly both in travel time and waveform tomography. It means that the inversion has a better ray coverage, to produce a velocity image with better resolution.

\section{The algorithms}

We have made two developments in this paper. First, in travel time tomography, as the (horizontal) velocity and the anisotropic parameter have different sensitivities, we use a subspace inversion method to invert these two parameters simultaneously. Second, in waveform tomography, we include the $2 \mathrm{D}$ anisotropic parameter model in the waveform calculation, so that seismic waveform is better matched through the inversion.

Assuming the media be the elliptical anisotropy, the velocity variation with direction is defined as

$$
v(\theta)=v_{v}\left(1+\varepsilon \sin ^{2} \theta\right),
$$

where $v_{v}$ is the vertical component of $\mathrm{P}$-wave velocity, $\theta$ is the angle variation against the vertical axis, $\varepsilon$ is an anisotropic parameter,

$$
\varepsilon=\frac{v_{h}-v_{v}}{v_{v}},
$$

and $v_{h}$ is horizontal velocity when $\theta=\pi / 2$.

Considering the ray path through the $i$ th cell and given $\left(x_{i}, z_{i}\right)$ and $\left(x_{i+1}, z_{i+1}\right)$ the begin and end points of the ray segment, then

$$
\sin \theta=\frac{\Delta x}{\sqrt{\Delta x^{2}+\Delta z^{2}}},
$$

where $\Delta x=x_{i+1}-x_{i}, \Delta z=z_{i+1}-z_{i}$. The travel time of this ray segment can be represented as

$$
\Delta t=s \frac{\sqrt{\Delta x^{2}+\Delta z^{2}}}{\frac{1}{1+\varepsilon}\left(1+\frac{\varepsilon \Delta x^{2}}{\Delta x^{2}+\Delta z^{2}}\right)},
$$

where $s=1 / v_{h}$ is the horizontal slowness. The travel time along the entire ray path between a source-receive pair is

$$
T=\sum_{i} \Delta t_{i}(s, \varepsilon)
$$
is,

According to Fermat principle, the ray path is the path which has minimal travel time, that 


$$
\begin{aligned}
& \partial T / \partial x_{i}=0, \\
& \partial T / \partial z_{i}=0 .
\end{aligned}
$$

Ray-tracing through the anisotropic media is performed by solving system (6).

Travel time inversion solves the following linear system,

$$
\left[\mathbf{B}_{s}, \mathbf{B}_{\varepsilon}\right]\left(\begin{array}{c}
\Delta s \\
\Delta \boldsymbol{\varepsilon}
\end{array}\right)=[\Delta \mathbf{d}]
$$

where $\mathbf{B}_{s}$ and $\mathbf{B}_{\varepsilon}$ are Frechet matrices in which the $k$ th row and $j$ th column element are given by $\left[\mathbf{B}_{s}\right]_{k j}=\partial t_{k} / \partial s_{j}$ and $\left[\mathbf{B}_{\varepsilon}\right]_{k j}=\partial t_{k} / \partial \varepsilon_{j}$, respectively, $t_{k}$ is travel time of $k$ th ray path, and $\Delta \mathbf{d}$ is travel time data residual.

However, the two parameters, $s$ and $\varepsilon$, have different physical dimensions and different sensitivities in the inversion, which could cause bias in the solution. We adopt a subspace scheme to deal with the different functional dependences on the various parameter types in a balance way (Kennet et al. 1988, Kennet and Sambridge 1998). Following Wang and Houseman $(1994,1995)$, we explore the gradients with respect to different parameters, as the measurements of sensitivity, and partition them into different subspaces. Therefore, the inversion procedure for each iteration may be divided into following steps:

1) Perform ray-tracing to calculate $\mathbf{B}=\left[\mathbf{B}_{s}, \mathbf{B}_{\varepsilon}\right]$, based on the current model $\mathbf{m}=\left(\begin{array}{l}\mathbf{s} \\ \boldsymbol{\varepsilon}\end{array}\right)$.

$$
\mathbf{g}=\left[\mathbf{g}_{s}, \mathbf{g}_{\varepsilon}\right]=\mathbf{H} \cdot \mathbf{m}-\mathbf{B}^{T} \Delta \mathbf{d},
$$

where $\mathbf{H}$ is the Hessian matrix.

3) Normalize two class gradients $\hat{\mathbf{g}}_{s}=\mathbf{g}_{s} /\left\|\mathbf{g}_{s}\right\|$ and $\hat{\mathbf{g}}_{\varepsilon}=\mathbf{g}_{\varepsilon} /\left\|\mathbf{g}_{\varepsilon}\right\|$, to build a projection matrix

$$
\mathbf{A}=\left[\hat{\mathbf{g}}_{s}, \hat{\mathbf{g}}_{\varepsilon}\right]
$$

4) Calculate step length of two parameter classes by

$$
\mathbf{u}=-\left(\mathbf{A}^{T} \mathbf{H A}\right)^{-1} \mathbf{A}^{T} \mathbf{g} ;
$$

5) Update model with $\mathbf{m}^{(n+1)}=\mathbf{m}^{(n)}+\mathbf{A u}$, where $\mathrm{n}$ is the iteration index.

Considering the elliptic anisotropy, the wave number in the $z$ direction is defined as

$$
k_{z}=\frac{v_{h}}{v_{v}} \sqrt{\frac{\omega^{2}}{v_{h}^{2}}-k_{x}^{2}},
$$

where $\left(k_{x}, k_{z}\right)$ are the horizontal and vertical components of the wave number vector, and $\omega$ is the angular frequency. Multiplying both sides with the wavefield in the Fourier domain, $F\left(k_{x}, z, \omega\right)$,

$$
k_{z}^{2} F\left(k_{x}, z, \omega\right)=\frac{v_{h}^{2}}{v_{v}^{2}}\left(\frac{\omega^{2}}{v_{h}^{2}}-k_{x}^{2}\right) F\left(k_{x}, z, \omega\right) .
$$

applying an inverse Fourier transform with respect to $k_{z}$,

$$
\frac{d^{2} F\left(k_{x}, z, \omega\right)}{d z^{2}}=-\frac{v_{h}^{2}}{v_{v}^{2}}\left(\frac{\omega^{2}}{v_{h}^{2}}-k_{x}^{2}\right) F\left(k_{x}, z, \omega\right) .
$$

and an inverse Fourier transform with respect to $k_{x}$, yields 


$$
\frac{v_{v}^{2}}{v_{h}^{2}} \frac{d^{2} F}{d z^{2}}+\frac{d^{2} F}{d x^{2}}+\frac{\omega^{2}}{v_{h}^{2}} F(x, z, \omega)=0 .
$$

Finally, we obtain

$$
\frac{1}{(1+\varepsilon)^{2}} \frac{d^{2} F}{d z^{2}}+\frac{d^{2} F}{d x^{2}}+\frac{\omega^{2}}{v_{h}^{2}} F(x, z, \omega)=0 .
$$

Using this equation, a 2D anisotropy model $\varepsilon(x, z)$ can be included in the waveform simulation. It is in contrast to a constant shrink factor used in the previous publication to include the anisotropy effect (Pratt and Shipp 1999). For the details of waveform tomography implemented in the frequency domain, please refer to Wang and Rao (2006).

\section{Real data example}

The real data set was acquired from a pair of vertical boreholes. The distance between two boreholes is $192 \mathrm{~m}$. A string of 12 hydrophone receivers at 3 meter spacing was placed in the left hand side well. Small explosive charges were fired successively in the other borehole at 3 meters intervals. The triggering signal for the seismograph was obtained by wrapping a wire around the end of the detonator.

There are total of 111 shot positions at the left hand side well, and same number of receiver positions at the right hand side well, ranging from $942 \mathrm{~m}$ to $1275 \mathrm{~m}$. Here, the actual solution domain is from 0 to $192 \mathrm{~m}$ in the $x$ direction and 942 to $1275 \mathrm{~m}$ in the $z$ direction. In the tomography inversion, we divide it into $64 \times 111$ cells with cell size of $3 \mathrm{~m}$.

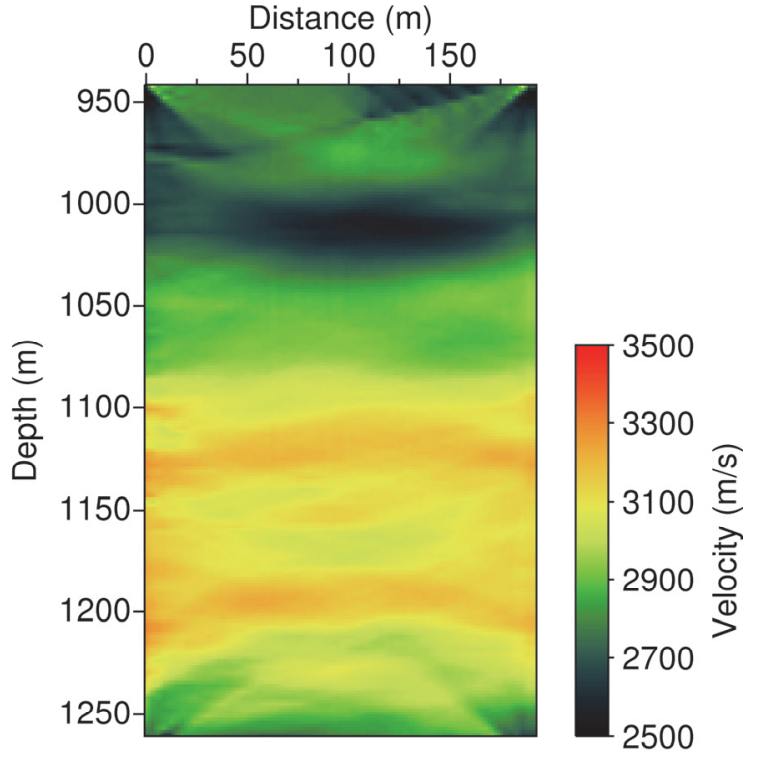

(a)

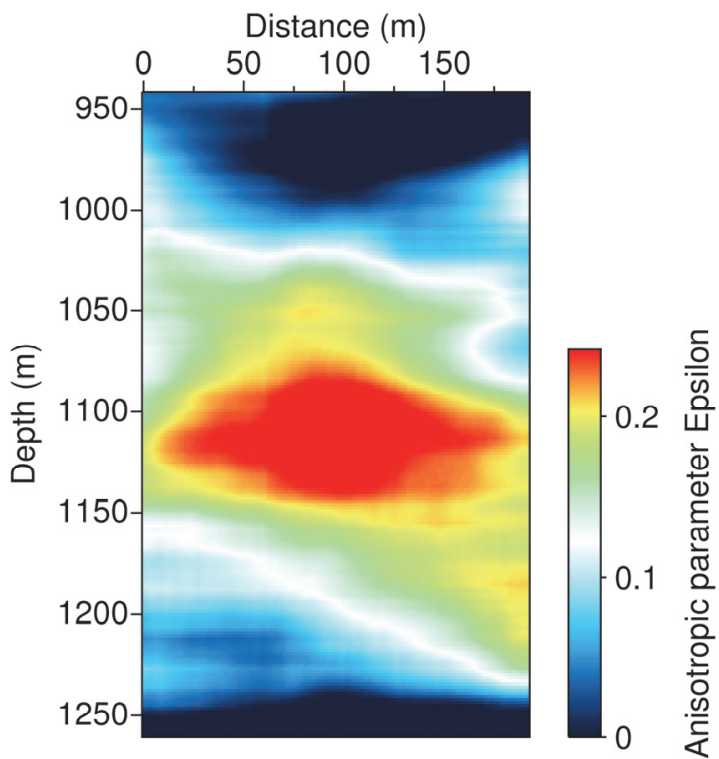

(b)

Figure 1. Travel time tomography for the anisotropic velocity. (a) Inverted (horizontal) velocity model. (b) Inverted anisotropy parameter $\varepsilon$ model.

Figure 1 displays the results of travel time tomography. The start velocity model for travel time tomography is generated by beck prorogation of first arrival times along straight ray paths linking source and receiver points. In Figure 1a, the horizontal velocity model, the layered structure has been reconstructed by travel time tomography, and some velocity anomalies have been shown clearly. In Figure $1 b$, the anisotropy parameter $(\varepsilon)$ model, the area with high anisotropy values (about 0.25 ), between 1075 and $1150 \mathrm{~m}$ in depth, corresponds to the complex layered sequences in the velocity model. 


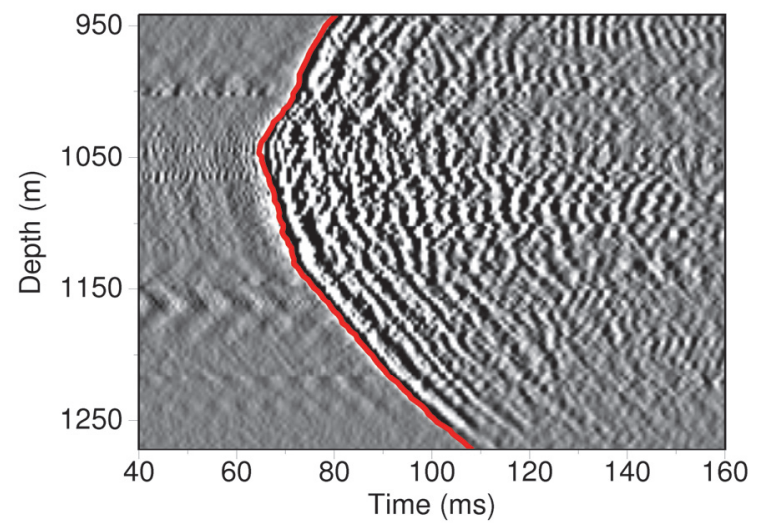

(a)

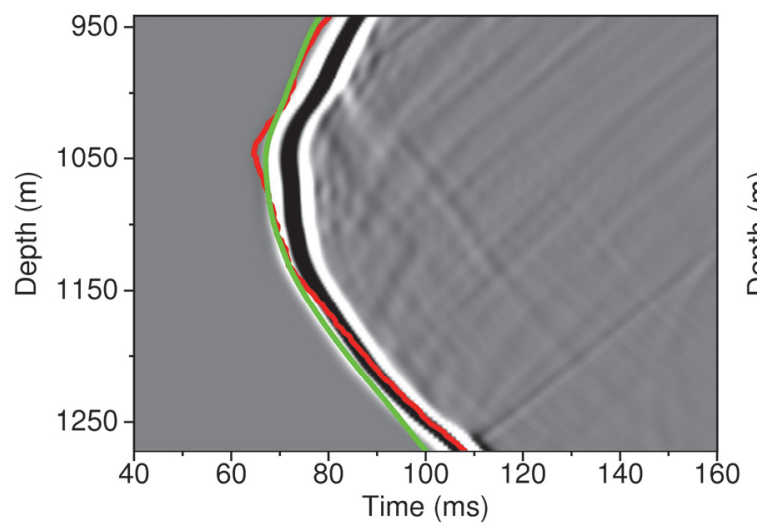

(b)

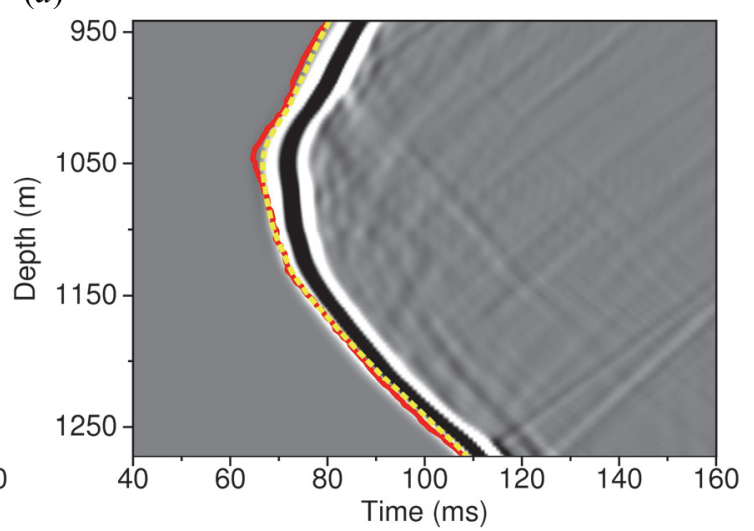

(c)

Figure 2. (a) An example shot gather of the real data set with source at $1050 \mathrm{~m}$ depth. The red curve is the first arrive time line picked from real data. (b) Modelled shot gather generated based only on the (horizontal) velocity model. The green curve is the first arrive time line of the synthetic without considering anisotropy effect. It has discrepancy from the red curve of the real data. (c) Modelled shot gather generated using both the (horizontal) velocity model and the anisotropy model. The yellow curve is the first arrive time line, estimated by traveltime tomography with considering anisotropy effect. It matches the real data curve.

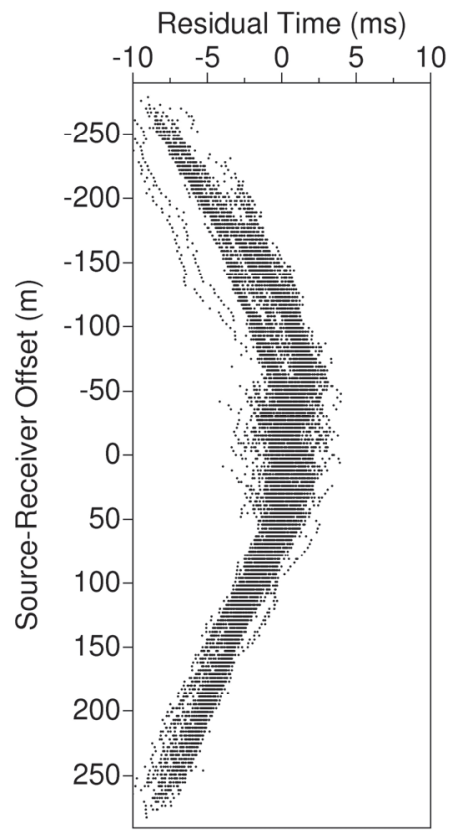

(a)

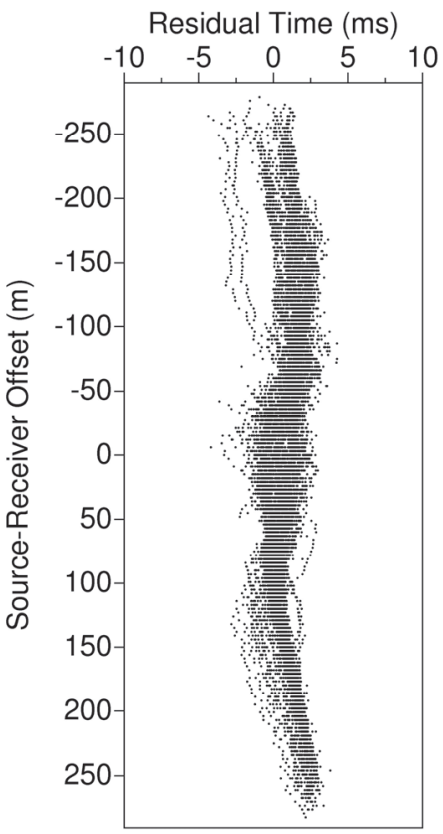

(b)

Figure 3. Predicted travel time residuals versus source-receiver offsets. (a) Estimated by traveltime tomography without considering the anisotropy effect. (b) With the consideration of the anisotropy effect. 
Figure $2 \mathrm{a}$ is an example shot gather, at depth of $1050 \mathrm{~m}$. Figure $2 \mathrm{~b}$ is the synthetic gather generated based on the velocity model alone, without considering the anisotropic effect. The modelled travel times can match near-offset traces, but have poor fitting to the far-offset first arrival times. In Figure 2c, however, the synthetic gather generated based on both the velocity model and the anisotropic parameter model, modelled first arrivals are almost overlapped with picked travel times, both in near and far offset traces.

Figure 3 shows the traveltime residuals versus source-receiver (vertical) offsets. If ignoring the anisotropic effect, the time residuals are between -2.5 and $2.5 \mathrm{~ms}$ in near offset traces, while in the far-offset area, the residuals reach $10 \mathrm{~ms}$. If taking account the anisotropic effect, the residuals are between -3 and $3 \mathrm{~ms}$ in both near and far offset traces.

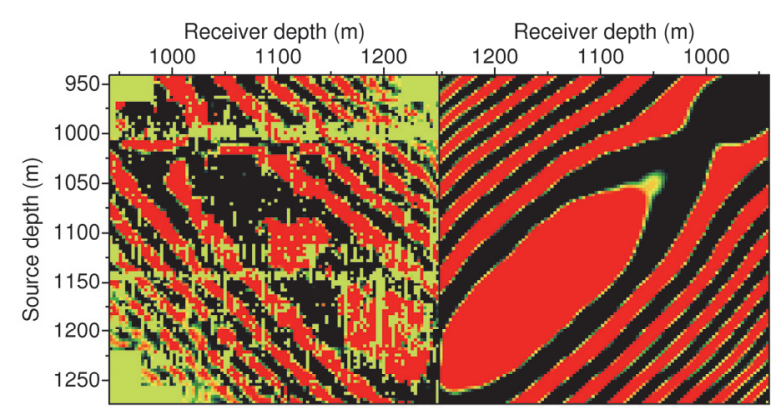

(a)

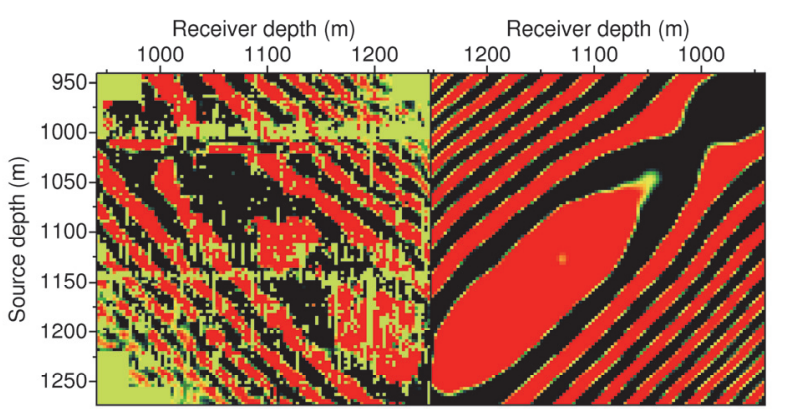

(b)

Figure 4. Comparison of (the real part of) data slices at frequency $113.3 \mathrm{~Hz}$. (a) The left half is the synthetic data slice generated from the inverted (horizontal) velocity model alone (without considering the anisotropy effect), and the other half is the mirror image of the observed data slice. $(b)$ The left half is the synthetic data slice generated based on both the (horizontal) velocity model and the anisotropy model, and the other half is the mirror image of the observed data slice.

As the waveform tomography that follows is implemented in the frequency domain, we closely compare the frequency-domain data slices (at frequency $113.3 \mathrm{~Hz}$ ) in a mirror imaging way, as shown in Figure 4: the left half is the synthetic data slice, and the other half is the mirror image of the observed data slice. The synthetic in Figure 4a is generated based only on the (horizontal) velocity model inverted from travel time tomography, and that in Figure $4 \mathrm{c}$ is based on both the (horizontal) velocity model and the anisotropic parameter model. Because all synthetic data are generated based on the travel time tomography result, we mainly focus on the major characteristics:

1) Comparing two synthetics, we can see that the banded structures between the offset -50 and $-250 \mathrm{~m}$ and 50 and $250 \mathrm{~m}$ are thinner, when the anisotropy effect is included.

2) The comparison between synthetic without anisotropy and the observed data, we can see a shift of those banded structures. But when the synthetic including anisotropy, the banded structures can well match the observed data.

These comparisons further confirm the existence of the anisotropy effect in the research area, because matched phase means that the more reasonable model is used in waveform simulation.

In waveform tomography, the anisotropic parameter model (Figure 1b) is included in waveform simulation, and the velocity model (Figure 1a) inverted from travel time tomography is used as the initial model for the iterative waveform tomography, preformed from low-frequency data to high-frequency data gradually. Comparing Figure 1a and 5, we see that waveform tomography has higher resolution than travel time tomography. Particularly, the sequential thin-layer structure, from 1100 to $1250 \mathrm{~m}$ in depth, has been shown sharply in Figure 5, while it is blurred in Figure 1a.

Waveform tomography is implemented by data matching in the frequency domain. The left column in Figure 6 is the observed data slices, arbitrarily selected at frequency $112 \mathrm{~Hz}$ (a), and $266 \mathrm{~Hz}$ (b). The right column is the estimated frequency slices in waveform tomography. 
In all source and receiver positions, the estimated data match the characters of the observed data, thanks to the anisotropic parameter model used in waveform simulation.

\section{Conclusions}

In crosshole seismic data, the anisotropy caused by fine layering has strong effect to far-offset data. We perform travel time tomography inverts for the horizontal velocity and the anisotropic parameter simultaneously, by using the subspace method. Considering the anisotropy parameter in travel time tomography results in a much better match in first arrival times between the synthetic and observation, particularly at far-offset part. We then include the anisotropy parameter model in waveform simulation, so that the far-offset energy can be used properly in waveform tomography. Consequently, modelled wave fronts match that in the observed shot gathers, and the phases of modelled frequency slices match well the observed data.

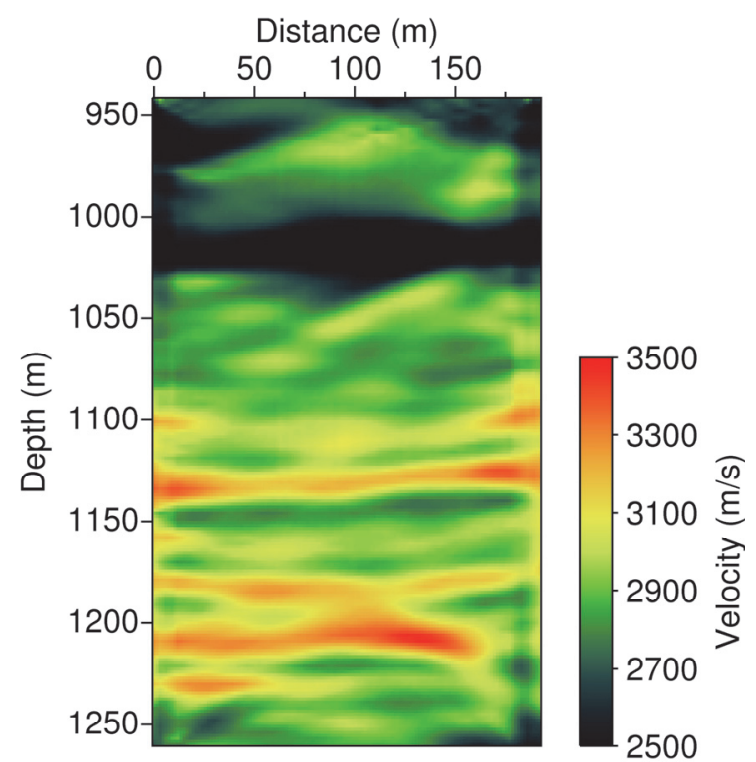

Figure 5. Reconstructed velocity model generated by waveform tomography. The initial velocity model for waveform inversion is given by travel time tomography (Figure 1a). The frequency components used in waveform tomography are from 110 to $300 \mathrm{~Hz}$.

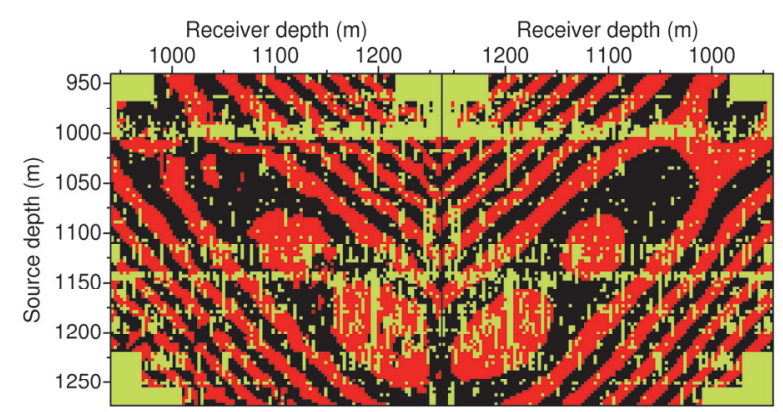

(a)

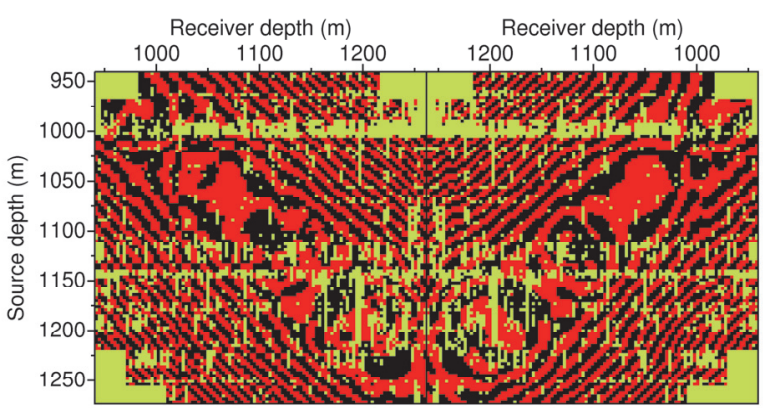

(b)

Figure 6. Comparison of frequency slices of the observed crosshole seismic data (left column) and synthetic data (right column) at $112 \mathrm{~Hz}(a)$ and $266 \mathrm{~Hz}(b)$. The synthetic data are generated based on the velocity model reconstructed by waveform tomography.

\section{Acknowledgements}

We are grateful to the sponsors of the Centre for Reservoir Geophysics, Imperial College London, for supporting this research. 


\section{References}

Chapman C H and Pratt R G 1992 Traveltime tomography in anisotropy media-I theory Geophys. J. Int. 109 1-19

Kennett B L N, Sambridge M S and Williamson P R 1988 Subspace methods for large inverse problems with multiple parameter classes Geophys. J. 94 237-47

Kennet B L N and Sambridge M 1998 Inversion for multiple parameter classes Geophys. J. Int. 135 $304-6$

Pratt R G and Chapman C H 1992 Traveltime tomography in anisotropy media-II Application Geophys. J. Int. 109 20-37

Pratt R G, McGaughey W J and Chapman C H 1993 Anisotropy velocity tomography: A case study in a near-surface rock mass Geophysics 58 1748-63

Pratt R G and Sams M S 1996 Reconciliation of crosshole seismic velocities with well information in a layered sedimentary environment Geophysics $61549-60$

Pratt R G and Shipp R M 1999 Seismic waveform inversion in the frequency domain, Part 2: Fault delineation in sediments using crosshole data Geophysics 64 902-14

Rao Y and Wang Y 2005 Crosshole seismic tomography: working solutions to issues in real data travel time inversion J. Geophys. Eng 2 139-46

Thomsen L 1986 Weak elastic anisotropy Geophysics 51 1954-66

Wang Y and Houseman G A 1994 Inversion of reflection seismic amplitude data for interface geometry Geophys. J. Int. 117 92-110

Wang Y and Houseman G A 1995 Tomographic inversion of reflection seismic amplitude data for velocity variation Geophys. J. Int. 123 355-72

Wang Y and Rao Y 2006 Crosshole seismic waveform tomography, I: Strategy for real data application Geophys. J. Int. 166 1237-48

Zhou B, Greenhalgh S and Green A 2008 Nonlinear traveltime inversion scheme for crosshole seismic tomography in tilted transversely isotropic media Geophysics 73 D17-33 